\title{
Differentially expressed miRNAs in oxygen-induced retinopathy newborn mouse models
}

\author{
YUNPENG WANG ${ }^{1 *}$, SUYING WU $^{2 *}$, YANG YANG $^{3}$, FEN PENG $^{2}$, QINTAO LI $^{2}$, PENG TIAN $^{2}$, ERYING XIANG $^{2}$, \\ HONGLU LIANG ${ }^{3}$, BEIBEI WANG ${ }^{3}$, XIAOYU ZHOU ${ }^{3}$, HUA HUANG $^{2}$ and XIAOGUANG ZHOU ${ }^{3}$ \\ ${ }^{1}$ Department of Neonatology, Nanshan People's Hospital, Affiliated to Guangdong Medical University, Shenzhen, \\ Guangdong 518052; ${ }^{2}$ Department of Neonatology, University Hospital of Hubei Minzu University, \\ Enshi, Hubei 445000; ${ }^{3}$ Department of Neonatology, Children's Hospital of Nanjing Medical University, \\ Nanjing, Jiangsu 210008, P.R. China
}

Received October 10, 2015; Accepted September 8, 2016

DOI: $10.3892 / \mathrm{mmr} .2016 .5993$

\begin{abstract}
The present study aimed to identify microRNAs (miRNAs) involved in regulating retinal neovascularization and retinopathy of prematurity (ROP). A total of 80 healthy C57BL/6 neonatal mice were randomly divided into the oxygen-induced retinopathy (OIR) group $(n=40)$, in which 7 -day-old mice were maintained in $75 \%$ oxygen conditions for 5 days, or the control group $(n=40)$. Following collection of retinal tissue, retinal angiography and hematoxylin and eosin (H\&E) staining were performed. Total RNA was also extracted from retinal tissue, and miRNA microarrays and reverse transcription-quantitative polymerase chain reaction (RT-qPCR) were performed to identify differentially expressed miRNAs in the two groups. Retinal angiography and H\&E staining revealed damage to retinas in the OIR group. Compared with the control group, 67 miRNAs were differentially expressed in the OIR group, of which 34 were upregulated and 33 were downregulated. Of these differentially expressed miRNAs, 32 exhibited a fold change $\geq 2$, of which 21 were upregulated and 11 were downregulated. The results of RT-qPCR for miR-130a-3p and miR-5107-5p were in accordance with those of the miRNA microarray. The newly identified miRNAs may be important in the development of
\end{abstract}

Correspondence to: Professor Hua Huang, Department of Neonatology, University Hospital of Hubei Minzu University, 2 Wufengshan Road, Enshi, Hubei 445000, P.R. China

E-mail: hhyjxx@sohu.com

Professor Xiaoguang Zhou, Department of Neonatology, Children's Hospital of Nanjing Medical University, 72 Guangzhou Road, Nanjing, Jiangsu 210008, P.R. China

E-mail: gzzhouxg@163.com

${ }^{*}$ Contributed equally

Key words: retinopathy of prematurity, microRNA, mice, oxygen-induced retinopathy, animal model
ROP, and may provide a basis for future research into the mechanisms of ROP.

\section{Introduction}

Retinopathy of prematurity (ROP) is a vascular proliferative disease that primarily occurs in preterm infants, causing disorganized growth of retinal blood vessels, which results in scarring, retinal detachment and occasionally blindness (1). ROP is associated with low gestational age and birth weight; although advances in neonatal care have resulted in improved survival rates for premature infants, the incidence of ROP has increased $(2,3)$. Numerous studies have been undertaken to examine the pathophysiological mechanisms of $\operatorname{ROP}(4,5)$; however, these currently remain unclear.

Animal models of oxygen-induced retinopathy (OIR) have previously been used to reveal mechanisms of ROP development, including oxidant stress, the functions of growth factors including insulin-like growth factor (IGF) and vascular endothelial growth factor (VEGF), and structure-functional anomalies $(6,7)$. Bioinformatic strategies have also been widely used to investigate ROP mechanisms; a study by Sato et al (8) investigated gene-expression profiles in murine oxygen-induced retinopathy as early as 2009 .

microRNAs (miRNAs) are small non-coding RNAs of 18-26 nucleotides in length that regulate gene expression through sequence-specific base pairing with target mRNAs. Numerous miRNAs have been identified through molecular cloning and bioinformatics strategies $(9,10)$. miRNAs generally inhibit mRNA translation of their target genes (11), and have been reported to be important in biological processes including vascular angiogenesis, cell growth, embryonic development, cell proliferation, tissue differentiation and apoptosis (12-15).

It is well documented that miRNAs regulate vascular angiogenesis (16) and the regulation of VEGF, IGF-2 and hypoxia-inducible factor $1 \alpha$ (HIF-1 $\alpha$ ) by miRNA (miR)-126 is associated with retinal vascular angiogenesis under conditions of OIR (17). However, whether other miRNAs are involved in the pathological process of angiogenesis in OIR conditions currently remains unclear. Therefore, the present 
Table I. Context sequences of the probes of mmu-miR-130a-3p, mmu-miR-5107-5p and the internal control U6 snRNA used in the present study.

Assay name

Context sequence $\left(5^{\prime}-3^{\prime}\right)$

mmu-miR-130a

mmu-miR-5107

U6 snRNA

\author{
CAGUGCAAUGUUAAAAGGGCAU \\ UGGGCAGAGGAGGCAGGGACA \\ GTGCTCGCTTCGGCAGCACATATACTAAAATTGGAACGATAC \\ AGAGAAGATTAGCATGGCCCCTGCGCAAGGATGACACGCAA \\ ATTCGTGAAGCGTTCCATATTTT
}

hsa, Homo sapiens; miR, microRNA; mmu, Mus musculus; snRNA, spliceosomal RNA.

Table II. RNA purity from retinal tissue RNA extractions.

\begin{tabular}{lcccccc}
\hline Sample group & $\begin{array}{c}\text { Concentration } \\
(\mu \mathrm{g} / \mu \mathrm{l})\end{array}$ & $\begin{array}{c}\mathrm{UV} \\
\mathrm{A} 260 / \mathrm{A} 280\end{array}$ & $\begin{array}{c}\mathrm{UV} \\
\mathrm{A} 260 / \mathrm{A} 230\end{array}$ & $\begin{array}{c}\text { Quantity } \\
(\mu \mathrm{g})\end{array}$ & $\begin{array}{c}\text { Bioanalyzer } \\
\text { result }\end{array}$ \\
\hline Oxygen-induced retinopathy & 0.25 & 2.00 & 2.21 & 10.72 & 2.5 & Pass \\
Control & 0.34 & 2.03 & 2.28 & 14.59 & 2.4 & Pass \\
\hline
\end{tabular}

${ }^{a}$ The $28 \mathrm{~S} / 18 \mathrm{~S}$ ribosomal RNA ratio was used to assess the quality of the RNA. Ratios $>2$ indicate that the total RNA is high quality.

study aimed to screen for miRNA candidates affecting retinal neovascularization using an OIR mouse model.

\section{Materials and methods}

Establishment of oxygen-induced retinopathy mouse model. A total of 80 (36 female; 44 male) 7-day-old healthy C57BL/6 neonatal mice (weight, $3 \pm 0.3 \mathrm{~g}$ ) were provided by the Animal Center of Nanjing University (Nanjing, China). All procedures in the study followed the protocols approved by the Animal Care and Use Committees of Nanjing Medical University (Nanjing, China). The mice were maintained in specific-pathogen-free conditions at the Animal Center of Nanjing Medical University. The mice were reared in a clean grade animal room, at $23 \pm 2{ }^{\circ} \mathrm{C}$ and $50 \pm 10 \%$ humidity. The light did not exceed 350 lux and the light/dark cycle was 12-h. Food, water and bedding were replaced every two days. The oxygen concentration was monitored with an oxygen concentration detector. The mice were randomly divided into the OIR group $(\mathrm{n}=40)$ or the control group $(\mathrm{n}=40)$. For OIR modeling, 7 -day-old mice were maintained in $75 \%$ oxygen condition for 5 days, then in normal room air for a further 5 days. Mice in the control group were maintained in normal room air for the duration of the experiment. The present study was approved by the ethics review board of Nanjing Children's Hospital of Nanjing Medical University.

Retinal angiography. On postnatal day 17 (P17), $50 \mathrm{mg}$ high molecular weight fluorescein isothiocyanate dextrans (catalog no. 75005; Sigma-Aldrich; Merck Millipore, Darmstadt, Germany) were dissolved in $1 \mathrm{ml}$ phosphate buffer containing $4 \%$ polyformaldehyde and directly perfused through the left ventricle of the heart following an intraperitoneal injection of $10 \%$ chloral hydrate $(3 \mathrm{ml} / \mathrm{kg}$; Sigma-Aldrich;
Merck Millipore). The eyes were then enucleated and fixed with $4 \%$ paraformaldehyde at $4{ }^{\circ} \mathrm{C}$ for $2 \mathrm{~h}$. The retina was separated and mounted on a microscope slide. The vasculature was examined under a fluorescent microscope (Nikon Corporation, Tokyo, Japan) at an original magnification of $\mathrm{x} 40$. In total, 30 sections were counted, and 3 fields of view were assessed for each slide.

Hematoxylin andeosin(HE) staining.Paraformaldehyde-fixed retinal tissue was embedded in paraffin and sliced into $6 \mu \mathrm{m}$ sections. The sections were stained with $\mathrm{HE}$ and examined by routine light microscopy (Olympus Corporation, Tokyo, Japan). Briefly, sections were dried, followed by xylene dewaxing and rehydration with decreasing concentrations of alcohol. The slides were then stained with hematoxylin for $15 \mathrm{~min}$ and differentiated with hydrochloric acid alcohol for $30 \mathrm{sec}$. Following staining with $1 \%$ ammonia, sections were stained with $1 \%$ eosin for $2 \mathrm{~min}$, followed by dehydration in alcohol and mounting. The number of vascular endothelial cells with nuclei breaking through the internal limiting membrane (ILM) was counted. In total, 30 sections were counted, and 3 fields of view were assessed for each slide.

Extraction and quantification of total RNA. For total RNA extraction, retinal tissue was frozen at $-80^{\circ} \mathrm{C}$ and crushed using a BioPulverizer (Aoran Technology Ltd., Shanghai, China). Homogenization was performed by adding $1 \mathrm{ml}$ TRIzol $^{\circledR}$ reagent (Invitrogen; Thermo Fisher Scientific, Inc., Waltham, MA, USA) to the tissue fragments, according to the manufacturer's protocol. Extracted RNA was dissolved in RNAse-free water and incubated at $60^{\circ} \mathrm{C}$ for $10 \mathrm{~min}$.

Absorbance at 260, 280 and $230 \mathrm{~nm}$ was measured using an ultraviolet spectrophotometer (NanoDrop Technologies; Thermo Fisher Scientific, Inc., Wilmington, DE, USA). The 
purity of the extracted RNAs was determined by calculating the A260/A280 and A260/A230 ratios.

miRNA microarray. Following isolation of total RNA from retinal tissue, microarray was performed by LC Sciences (Houston, TX, USA). Each detection probe on the microfluidic chips consisted of a chemically modified nucleotide coding segment complementary to target a total of 25,141 miRNAs (from miRBase version 19.0, www.mirbase.org/) or other RNA (control or customer-defined sequences) and a spacer segment of polyethylene glycol to extend the coding segment away from the substrate. The detection probes were made by in situ synthesis using photogenerated reagent chemistry. The hybridization melting temperatures were balanced by chemical modifications of the detection probes. Hybridization used $100 \mu \mathrm{l} 6 \mathrm{X}$ saline sodium phosphate EDTA buffer $(0.90 \mathrm{M} \mathrm{NaCl}$, $60 \mathrm{mM} \mathrm{Na} \mathrm{HPO}_{4}, 6 \mathrm{mM}$ ethylenediaminetetraacetic acid, $\mathrm{pH}$ 6.8) containing $25 \%$ formamide at $34^{\circ} \mathrm{C}$. Following RNA hybridization, tag-conjugating Cyanine 3 dye was circulated through the microfluidic chip at $34^{\circ} \mathrm{C}$ overnight. Fluorescence images were collected using a GenePix 4000B microarray scanner (Molecular Devices LLC, Sunnyvale, CA, USA) and visualized using Array-Pro image analysis software version 6.3 (Media Cybernetics, Inc., Rockville, MD, USA). Data were analyzed by subtracting the background, and then normalizing the signals using a LOWESS filter (locally-weighted regression).

Following normalization, differentially expressed miRNAs were identified through fold-change filtering (fold change $\geq 2$ ). Hierarchical clustering was performed using $\mathrm{MeV}$ software version 4.6 (The Institute for Genomic Research, J. Craig Venter Institute, Rockville, MA, USA).

Reverse transcription-quantitative polymerase chain reaction $(R T-q P C R)$. Following isolation of total RNA, $1 \mu \mathrm{g}$ total RNA was used for RT using the TaqMan ${ }^{\circledR}$ MicroRNA Reverse Transcription kit (Applied Biosystems; Thermo Fisher Scientific, Inc.) according to the manufacturer's protocol. The cDNA obtained was used for qPCR, with probes for Mus musculus (mmu)-miR-130a-3p and mmu-miR-5107-5p, listed in Table I. The hot start method was used for fluorescent qPCR and the standard internal reference [U6 spliceosomal RNA (snRNA)] coupled with the control group was amplified for each sample. qPCR was performed in a $20-\mu 1$ reaction mixture, with each tube containing $10 \mu \mathrm{l} 2 \mathrm{X}$ TaqMan Universal Master Mix II, $1 \mu 1$ TaqMan MicroRNA assays (Applied Biosystems; Thermo Fisher Scientific, Inc.), $1.33 \mu 1$ cDNA template and $7.67 \mu 1$ RNase-free water. The cycling conditions were as follows: An initial denaturation step at $50^{\circ} \mathrm{C}$ for $2 \mathrm{~min}$ and $95^{\circ} \mathrm{C}$ for $2 \mathrm{~min}$, followed by 40 cycles of denaturation at $95^{\circ} \mathrm{C}$ for $15 \mathrm{sec}$, and annealing/extension at $60^{\circ} \mathrm{C}$ for $1 \mathrm{~min}$. The U6 snRNA was used as an internal control for normalization. The relative quantitative (RQ) value, obtained using the $2^{-\Delta \Delta \mathrm{Cq}}$ method (18), was used for statistical analysis. Briefly, the cycle threshold $(\mathrm{Cq})$ values were calculated using automatic baseline settings. The $\Delta \mathrm{Cq}$ value between internal control $\mathrm{U} 6$ and the corresponding $\mathrm{Cq}$ value for the target gene was used for the relative quantification of miRNA expression.

Statistical analysis. Statistical analyses were performed using SPSS version 13.0 (SPSS, Inc., Chicago, IL, USA). Data were
Table III. Differentially expressed microRNAs upregulated ( $>2$-fold) in the oxygen-induced retinopathy group compared with the control group, listed and arranged according to magnitude of change.

\begin{tabular}{lrc}
\hline Name & Fold-change & P-value \\
\hline mmu-miR-1907 & 14.389 & 0.000108 \\
mmu-miR-3970 & 12.300 & 0.000069 \\
mmu-miR-5107-5p & 9.714 & 0.000632 \\
mmu-miR-133b-5p & 7.753 & 0.000973 \\
mmu-miR-3962 & 7.068 & 0.000009 \\
mmu-miR-145b & 5.077 & 0.000924 \\
mmu-miR-3072-5p & 3.690 & 0.002920 \\
mmu-miR-129-2-3p & 3.074 & 0.001340 \\
mmu-miR-714 & 3.000 & 0.004800 \\
mmu-miR-29c-3p & 2.833 & 0.001420 \\
mmu-miR-145a-5p & 2.500 & 0.003340 \\
mmu-miR-100-5p & 2.425 & 0.000081 \\
mmu-miR-3107-5p & 2.385 & 0.005480 \\
mmu-miR-30c-1-3p & 2.172 & 0.004510 \\
mmu-miR-96-5p & 2.157 & 0.009780 \\
mmu-miR-6236 & 2.148 & 0.006500 \\
mmu-miR-3473b & 2.122 & 0.005810 \\
mmu-miR-214-3p & 2.093 & 0.006740 \\
mmu-miR-9-5p & 2.089 & 0.000624 \\
mmu-miR-181d-5p & 2.070 & 0.002950 \\
mmu-miR-3473e & 2.045 & 0.005690 \\
\hline
\end{tabular}

mmu, Mus musculus; miR, microRNA.

Table IV. Differentially expressed microRNAs downregulated ( $>2$-fold) in the oxygen-induced retinopathy group compared with the control group, listed and arranged according to magnitude of change.

\begin{tabular}{lcc}
\hline Name & Fold-change & P-value \\
\hline mmu-miR-495-3p & 3.354 & 0.000002 \\
mmu-miR-677-3p & 3.255 & 0.000206 \\
mmu-miR-let-7d-3p & 2.606 & 0.002650 \\
mmu-miR-328-3p & 2.465 & 0.001420 \\
mmu-miR-301a-3p & 2.462 & 0.003470 \\
mmu-miR-128-3p & 2.149 & 0.002440 \\
mmu-miR-872-5p & 2.076 & 0.008250 \\
mmu-miR-181c-5p & 2.070 & 0.006170 \\
mmu-miR-744-5p & 2.065 & 0.004720 \\
mmu-miR-130a-3p & 2.000 & 0.000412 \\
mmu-miR-377-3p & 2.000 & 0.006950
\end{tabular}

mmu, Mus musculus; miR, microRNA.

expressed as the mean \pm standard deviation. Comparisons of retinal endothelial cell nucleus between the OIR and control groups were performed using the means t-test. RT-qPCR 
A

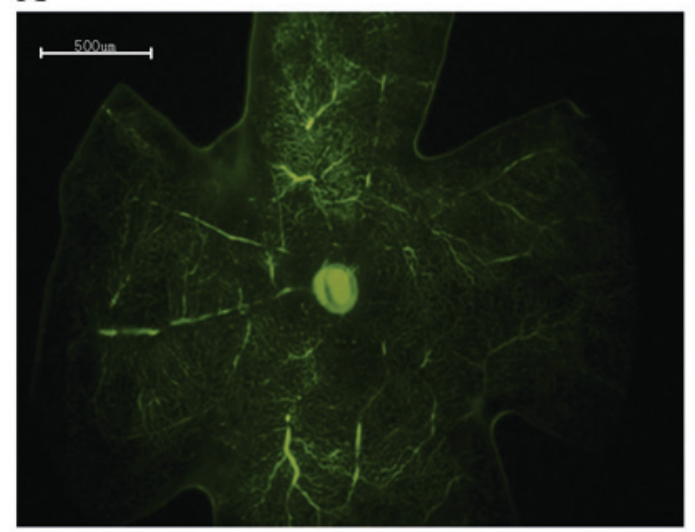

B

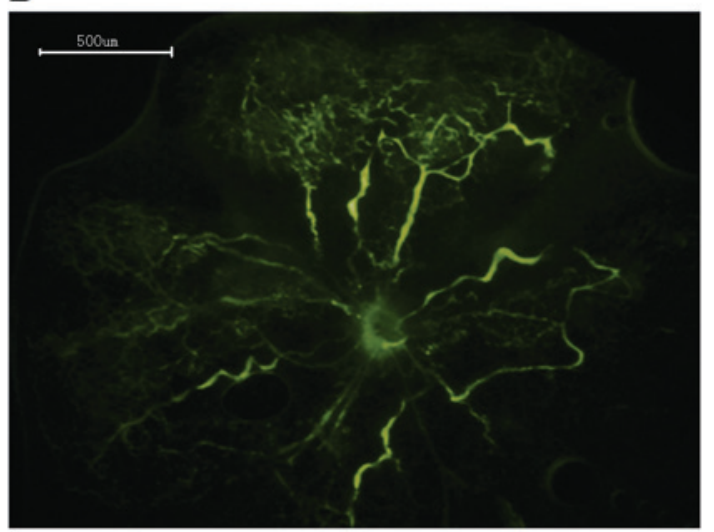

Figure 1. Retinal angiography with high-molecular-weight fluorescein-dextran. (A) Retina of the control group. No neovascularization was observed in the peripheral perfusion area. (B) Retina of the oxygen-induced retinopathy group. Decreased vascular plexus and vascular tortuosity was visible in the peripheral perfusion area. Original magnification, $\mathrm{x} 40$; scale bars $=500 \mu \mathrm{m}$.

A

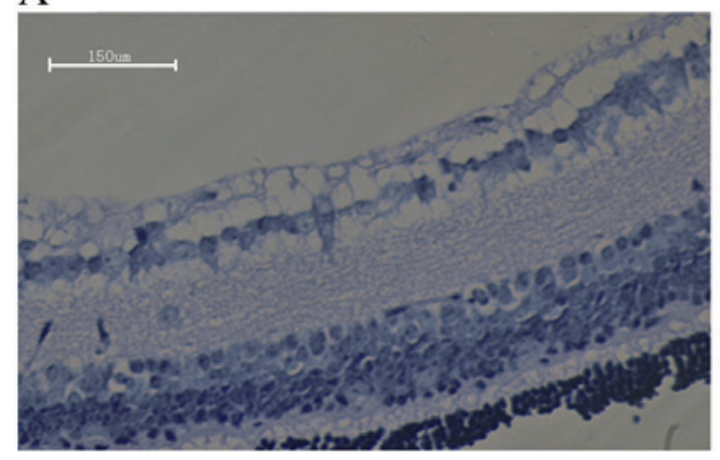

B

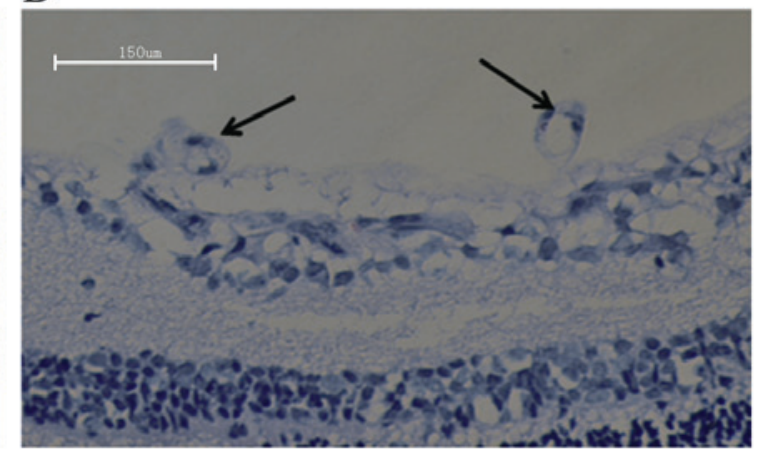

Figure 2. Hematoxylin and eosin staining of (A) control group and (B) oxygen-induced retinopathy group retinas. Arrows indicate the enlarged intraretinal vessel profiles. Original magnification, $\mathrm{x} 400$; scale bar $=150 \mu \mathrm{m}$.

data were evaluated by one-way analysis of variance and independent sample t-test. $\mathrm{P}<0.05$ was considered to indicate a statistically significant difference. To evaluate miRNA expression profiles, $\mathrm{t}$-tests were performed, and $\mathrm{P}<0.01$ was considered to indicate a statistically significant difference.

\section{Results}

Resultofretinalangiography.Retinalangiographywas performed to detect differences between the control and OIR groups, and thus determine whether the murine model was successfully established. The retinal vasculature of the control group extended from the optic nerve to the periphery, with the vessels forming a fine radial branching pattern (Fig. 1A). The retinal vascular pattern in the OIR group was characterized by decreased central perfusion, which was particularly notable in the central retina (Fig. 1B). The murine OIR model was, therefore, successfully established.

Histological changes. Histology was conducted to examine differences in retinal tissue between the control and OIR groups. Vascular cells in the control group did not extend from the internal limiting membrane into the vitreous (Fig. 2A), while vascular cells in the OIR group extended from the internal limiting membrane into the vitreous (Fig. 2B). The degree of hyperoxia-induced neovascularization was quantified by counting the number of vascular cell nuclei on the vitreal side of the internal limiting membrane of the retina. There was an average of $0.89 \pm 0.71$ nuclei extending into the internal limiting membrane in the control group, compared with $30.17 \pm 7.43$ nuclei in the OIR group $(\mathrm{P}=0.00006$; Fig. 3$)$. The retinal tissue in the OIR model was, therefore demonstrated to be damaged, further confirming the successful establishment of the OIR model.

Quality assessment of total retinal RNA. In order to determine the quality of the extracted retinal tissue RNA, total RNA quality assessment was performed. RNA with A260/A280 ratio close to 2.0 is regarded as pure RNA: A260/A280 ratio $<1.8$ indicates sample contamination, while a ratio $>2.0$ suggests RNA hydrolysis. An A260/A280 ratio in the range of 1.8-2.1 is acceptable. In addition, for pure RNA samples, the A260/A230 ratio should be $>1.8$. As presented in Table II, the A260/A280 ratios of the extracted RNA were $\sim 2.0$ and the 260/A230 ratios were all $>2.2$. The quality of the extracted RNA was therefore good.

miRNA expression profile. To examine changes in miRNA expression levels in mice in the OIR group, a microarray 


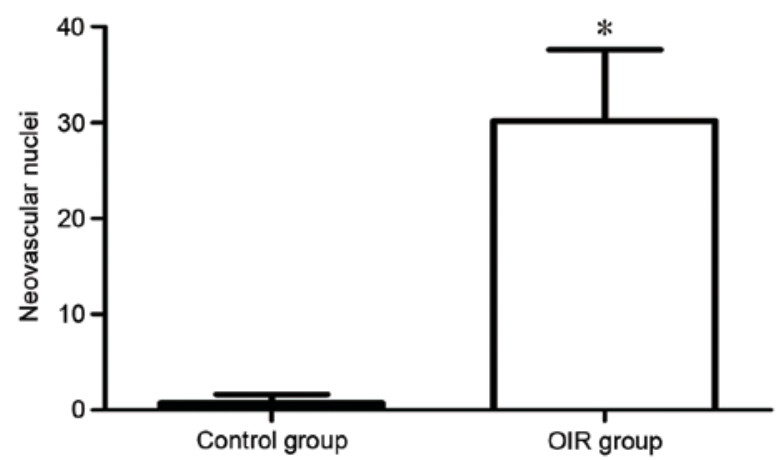

Figure 3. Quantification of proliferative neovascular response to OIR. The total number of vascular nuclei extending from the internal limiting membrane into the vitreous per section was counted. Values are presented as the mean number of nuclei \pm standard deviation. ${ }^{*} \mathrm{P}<0.0001$ vs. control group. OIR, oxygen-induced retinopathy.

analysis was performed to evaluate miRNA expression profiles. There were 67 differentially expressed miRNAs in the OIR group compared with the control group, of which, 34 were upregulated and 33 were downregulated. There were 32 differentially expressed miRNAs with a fold change $\geq 2.0$, of which, 21 were upregulated (Table III) and 11 were downregulated (Table IV). Of these miRNAs, 6 miRNAs that were upregulated in the OIR group compared with the control group (miR-1907, miR-3970, miR-5107-5p, miR-133b-5p, miR-3962 and miR-145b; Table III) and 11 miRNAs that were downregulated in the OIR group compared with the control group (miR-495-3p, miR-677-3p, miR-let-7d-3p, miR-328-3p, miR-301a-3p, miR-128-3p, miR-872-5p, miR-181c-5p, miR-744-5p, miR-130a-3p and miR-377-3p; Table IV) were statistically significantly different $(\mathrm{P}<0.05)$, with a $\geq 2$-fold change. Of the upregulated miRNAs in the OIR group, mmu-miR-1907 had the greatest fold-change compared with the control group, and of the downregulated miRNAs, mmu-miR-495-3p had the greatest difference compared with the control group. Hierarchical clustering revealed distinguishable miRNA expression profiles between the two groups, with $\mathrm{S} 01$ as the control group and S03 as the experimental group (Fig. 4). Thus, modulation of oxygen conditions resulted in differential miRNA expression.

Confirmation of differentially expressed miRNAs by RT-qPCR . To validate the differential expression profiles of miRNAs indicated by microarray analysis, RT-qPCR analysis of miRNAs was performed for mmu-miR-130a-3p and mmu-miR-5107-5p. These miRNAs were selected for confirmatory testing by RT-qPCR as they exhibited large fold-changes, large mean values and small P-values. The relative expression level of mmu-miR-130a-3p was significantly lower in the OIR group compared with the control group ( $\mathrm{P}=0.00239$; Fig. $5 \mathrm{~A})$, while that of mmu-miR-5107-5p was significantly higher in the OIR group compared with the control group ( $\mathrm{P}=0.0352$; Fig. 5B). The data from this confirmatory RT-qPCR analysis was, therefore, consistent with that of the microarray analysis.

\section{Discussion}

In the present study, a mouse model of OIR was constructed and subsequently used to identify differentially expressed

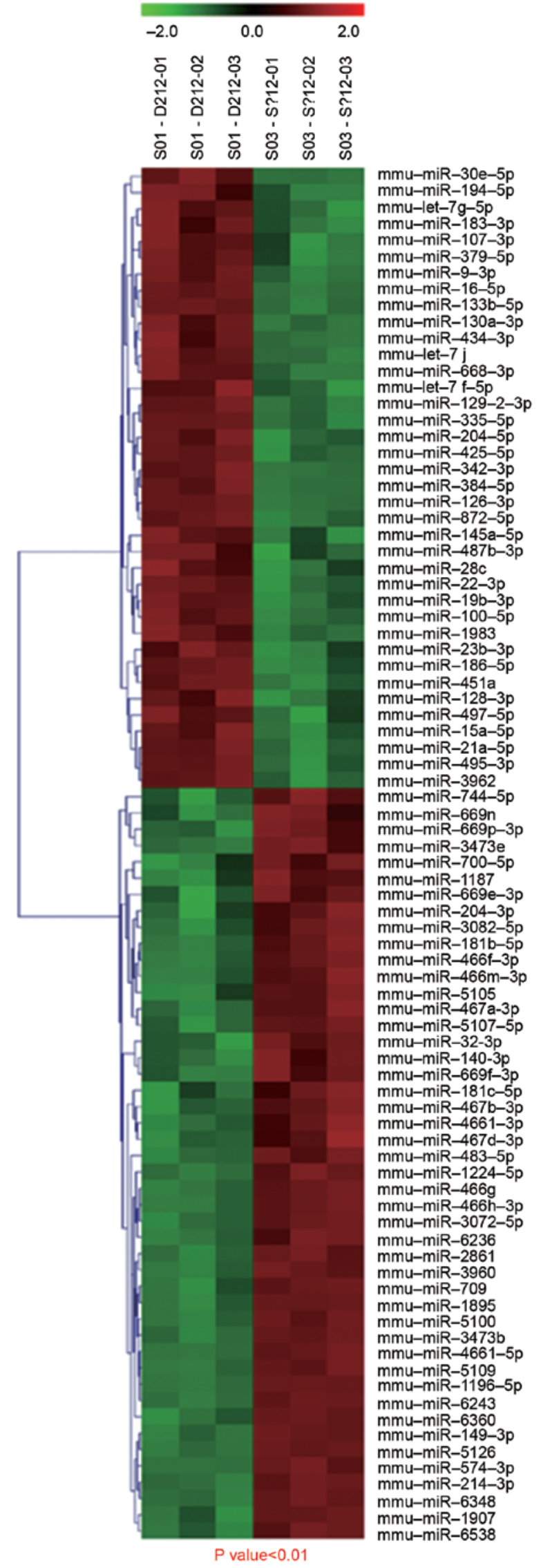

Figure 4. Hierarchical clustering of miRNAs in the control (S01) and oxygen-induced retinopathy (S03) groups. Two-way hierarchical cluster heat map presents all significantly expressed miRNAs: Each row displays the relative expression level of a single miRNA; each column displays the expression level of a single sample. Red, high relative expression; green, low relative expression; and black, zero. mmu, Mus musculus; miR, microRNA. 
A

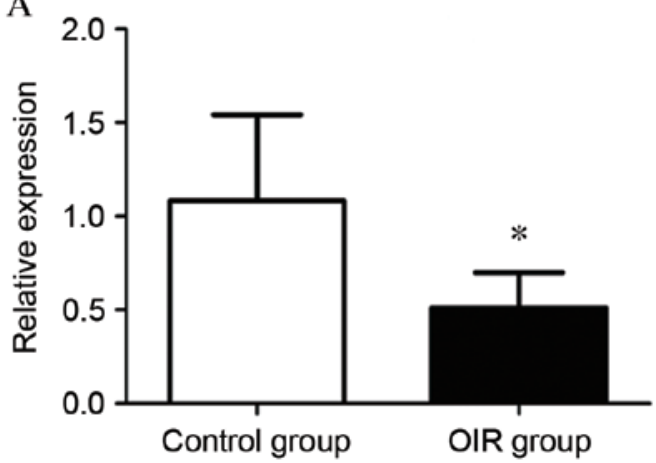

B

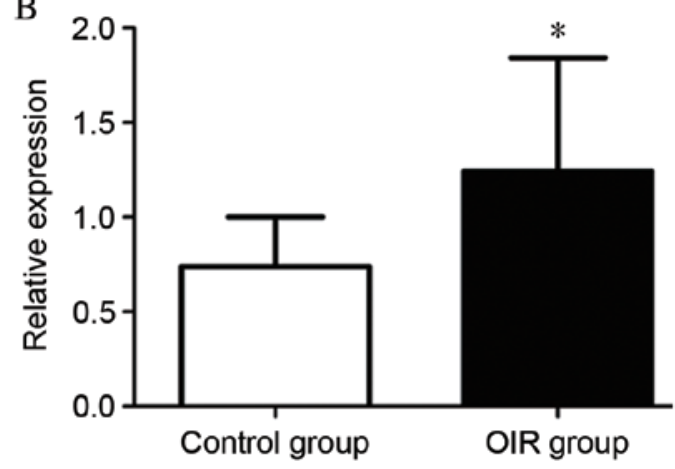

Figure 5. Verification of miRNA differential expression. Expression levels of (A) mmu-miR-130a-3p and (B) mmu-miR-5107-5p identified by miRNA microarray were examined by reverse transcription-quantitative polymerase chain reaction. Data are presented as the mean \pm standard deviation of one representative experiment and three independent experiments per sample $(\mathrm{n}=40)$ were performed for each miRNA. "P<0.05 vs. control group. miRNA, microRNA; mmu, Mus musculus; OIR, oxygen-induced retinopathy.

miRNAs via microarray. Two of the differentially expressed miRNAs identified by microarray were confirmed by RT-qPCR. Hyperoxia was, therefore, demonstrated to result in the dysregulation of miRNAs in murine retinal tissue, suggesting that miRNAs are important in the development and progression of oxygen-induced ROP.

The OIR mouse model was first developed in 1994 (7) and is commonly used for studies of retinal neovascularization in ROP and other vasculopathologies. Subsequent studies have demonstrated its utility in investigating the pathogenesis of retinal neovascularization and medical interventions for ROP and other retinal angiopathies $(8,19)$. The model has also proven to be reproducible and quantifiable, making it a favorable model for microarray analysis.

In the present study, a miRNA microarray was used to analyze miRNA expression profiles and to identify differentially expressed miRNAs in the retinal tissues of mice subjected to OIR: 67 differentially expressed miRNAs were identified in the OIR group compared with the control group. The functions of the majority of the upregulated miRNAs identified in the present study, including miR-1907, miR-3970, miR-5107-5p, miR-3962 and miR-145b, have not previously been reported. As a result, the effects of these miRNAs and potential applications of their functions require further investigations.

In the present study, the expression of miR-133b-5p was revealed to be up to 7.753 -fold higher in the OIR group compared with the control group, indicating a potential role for this miRNA in the development of ROP. miR-133b-5p regulates the expression of heat shock protein 70 (Hsp70) during gp120 V3 loop peptide-induced rat neuronal cell apoptosis (20). Hsp70 is a chaperone protein that inhibits key effectors of the apoptotic machinery at the pre- and postmitochondrial level (21). In addition, miR-133b, the precursor of miR-133b-5p, is downregulated in human osteosarcoma cells, resulting in increased apoptosis, and potentially acting as a tumor suppressor gene (22). The expression of miR-133b-5p may, therefore, regulate programmed cell apoptosis pathways and be involved in the development of ROP.

In contrast to the largely uncharacterized upregulated miRNAs, some of the downregulated miRNAs identified in the present study have been demonstrated to be involved in numerous diseases. The miR-1et-7 family is widely distributed in vertebrates and invertebrates, and participates in numerous biological processes, including reticulocytes (23), T-cell generation, angiogenesis (24) and the Fas-mediated apoptotic process in peripheral blood mononuclear cells $(25,26)$. It is, therefore, unsurprising that the expression of miR-let-7d-3p is decreased in a disease such as ROP, which is caused by abnormal blood vessel development. miR-328 expression has been demonstrated to be elevated in numerous lung adenocarcinoma subtypes, which may affect the degree of malignancy of tumor progression by inhibiting the proliferation of GBM cells in gliomas (27-30). A known target of miR-328 is RTP801, which is also a target of miR-181b-5p, which had an effect on the cancer stem cells fraction and neurosphere formation as surrogate marker (31). RTP801 expression is induced in the retina following hyperoxia treatment, while the development of retinopathy is significantly attenuated in an RTP801-knockout mouse model of ROP (32). These findings suggest important roles of miR-328 and miR-181b-5p in the pathogenesis of ROP by regulating the expression of RTP801.

The potential targets of other miRNAs are also related to ROP. One of the miR-872-5p targets is insulin-like growth factor 1, which acts upstream of retinal VEGF and is important in ROP (33). Angiopoietin 2, a potential target of miR-128-3p, induces endothelial cell apoptosis and is involved in ROP (31). miR-301a and miR-130a are miRNAs that have been suggested to bind to conserved sites of the peroxisome proliferator-activated receptor $\gamma$ (PPAR $\gamma$ ) 3' untranslated region (34). PPAR $\gamma$ is widely expressed in the eye, is involved in the pathogenesis of OIR (35), and provides a pharmacological target for treating ocular angiogenesis (36). Furthermore, miR-130a contributes to angiogenesis by inhibiting the expression of two antiangiogenic homeobox transcription factors, homeobox A5 (HOXA5) and mesenchyme homeobox 2 (MEOX2; also termed GAX) (11). However, further research is required to establish whether these miRNAs (miR-872-5p, miR-128-3p, miR-301a and miR-130a) regulate ROP development.

In summary, the present study has identified the differential expression of miRNAs in a murine model of OIR some of whose functions, or those of their known targets, are associated with the development of ROP. These findings are useful to understand the molecular basis of ROP and suggest that miRNAs are involved in the progression of ROP. However, 
further studies are required to confirm the involvement of the miRNAs identified in the present study in the development of ROP, and their molecular mechanisms.

\section{Acknowledgements}

The present study was supported by the National Natural Science Foundation of China (grant no. 81260106).

\section{References}

1. McGregor ML, Bremer DL, Cole C, McClead RE, Phelps DL, Fellows RR and Oden N; HOPE-ROP Multicenter Group. High Oxygen Percentage in Retinopathy of Prematurity study: Retinopathy of prematurity outcome in infants with prethreshold retinopathy of prematurity and oxygen saturation $>94 \%$ in room air: The high oxygen percentage in retinopathy of prematurity study. Pediatrics 110: 540-544, 2002.

2. Sola A, Chow L and Rogido M: Retinopathy of prematurity and oxygen therapy: A changing relationship. An Pediatr (Barc) 62: 48-63, 2005 (In spanish).

3. Chamney S, McGrory L, McCall E, Twaij S, Napier M, Rollins R, Marshall AH, Craig S and McLoone E: Treatment of retinopathy of prematurity in Northern Ireland, 2000-2011: A population-based study. J AAPOS 19: 223-227, 2015.

4. Levesque BM, Kalish LA, Winston AB, Parad RB, Hernandez-Diaz S, Phillips M, Zolit A, Morey J, Gupta M, Mammoto A, et al: Low urine vascular endothelial growth factor levels are associated with mechanical ventilation, bronchopulmonary dysplasia and retinopathy of prematurity. Neonatology 104: 56-64, 2013.

5. Jivabhai Patel S, Bany-Mohammed F, McNally L, Valencia GB, Lazzaro DR, Aranda JV and Beharry KD: Exogenous superoxide dismutase mimetic without scavenging $\mathrm{H}_{2} \mathrm{O}_{2}$ causes photoreceptor damage in a rat model for oxygen-induced retinopathy. Invest Ophthalmol Vis Sci 56: 1665-1677, 2015.

6. Penn JS, Tolman BL and Henry MM: Oxygen-induced retinopathy in the rat: Relationship of retinal nonperfusion to subsequent neovascularization. Invest Ophthalmol Vis Sci 35: 3429-3435, 1994.

7. Smith LE, Wesolowski E, McLellan A, Kostyk SK, D'Amato R, Sullivan R and D'Amore PA: Oxygen-induced retinopathy in the mouse. Invest Ophthalmol Vis Sci 35: 101-111, 1994.

8. Sato T, Kusaka S, Hashida N, Saishin Y, Fujikado T and Tano Y: Comprehensive gene-expression profile in murine oxygen-induced retinopathy. Br J Ophthalmol 93: 96-103, 2009.

9. Bentwich I, Avniel A, Karov Y, Aharonov R, Gilad S, Barad O, Barzilai A, Einat P, Einav U, Meiri E, et al: Identification of hundreds of conserved and nonconserved human microRNAs. Nat Genet 37: 766-770, 2005.

10. Xie M, Zhang S and Yu B: microRNA biogenesis, degradation and activity in plants. Cell Mol Life Sci 72: 87-99, 2015.

11. Doench JG and Sharp PA: Specificity of microRNA target selection in translational repression. Genes Dev 18: 504-511,2004

12. Kuhnert F, Mancuso MR, Hampton J, Stankunas K, Asano T, Chen CZ and Kuo CJ: Attribution of vascular phenotypes of the murine Egfl7 locus to the microRNA miR-126. Development 135 3989-3993, 2008.

13. Chen Y and Gorski DH: Regulation of angiogenesis through a microRNA (miR-130a) that down-regulates antiangiogenichomeobox genes GAX and HOXA5. Blood 111: 1217-1226, 2008.

14. Kuehbacher A, Urbich C, Zeiher AM and Dimmeler S: Role of Dicer and Drosha for endothelial microRNA expression and angiogenesis. Circ Res 101: 59-68, 2007.

15. Johnson CD, Esquela-Kerscher A, Stefani G, Byrom M, Kelnar K, Ovcharenko D, Wilson M, Wang X, Shelton J, Shingara J, et al: The let-7 microRNA represses cell proliferation pathways in human cells. Cancer Res 67: 7713-7722, 2007.

16. Shen J, Yang X, Xie B, Chen Y, Swaim M, Hackett SF and Campochiaro PA: MicroRNAs regulate ocular neovascularization. Mol Ther 16: 1208-1216, 2008.
17. Bai Y, Bai X, Wang Z, Zhang X, Ruan C and Miao J: MicroRNA-126 inhibits ischemia-induced retinal neovascularization via regulating angiogenic growth factors. Exp Mol Pathol 91: 471-477, 2011.

18. Livak KJ and Schmittgen TD: Analysis of relative gene expression data using real-time quantitative PCR and the 2(-Delta Delta C (T)) Method. Methods 25: 402-408, 2001.

19. Wang W, Li Z, Sato T and Oshima Y: Tenomodulin inhibits retinal neovascularization in a mouse model of oxygen-induced retinopathy. Int J Mol Sci 13: 15373-15386, 2012.

20. Xia C, Cai Y, Lin Y, Guan R, Xiao G and Yang J: MiR-133b-5p regulates the expression of the heat shock protein 70 during rat neuronal cell apoptosis induced by the gp120 V3 loop peptide. J Med Virol 88: 437-447, 2015.

21. Rérole AL, Jego G and Garrido C: Hsp70: Anti-apoptotic and tumorigenic protein. Methods 787: 205-230, 2011.

22. Zhao H, Li M, Li L, Yang X, Lan G and Zhang Y: MiR-133b is down-regulated in human osteosarcoma and inhibits osteosarcoma cells proliferation, migration, and invasion and promotes apoptosis. PLoS One 8: e83571, 2013.

23. Noh SJ, Miller SH, Lee YT, Goh SH, Marincola FM, Stroncek DF, Reed C, Wang E and Miller JL: Let-7 microRNAs are developmentally regulated in circulating human erythroid cells. J Transl Med 7: 98, 2009

24. Landskroner-Eiger S, Moneke I and Sessa WC: miRNAs as modulators of angiogenesis. Cold Spring Harb Perspect Med 3: a006643, 2013.

25. Vaz F, Hanenberg H, Schuster B, Barker K, Wiek C, Erven V, Neveling K, Endt D, Kesterton I, Autore F, et al: Mutation of the RAD51C gene in a Fanconi anemia-like disorder. Nat Genet 42: 406-409, 2010

26. Wang S, Tang Y, Cui H, Zhao X, Luo X, Pan W, Huang X and Shen N: Let-7/miR-98 regulate Fas and Fas-mediated apoptosis. Genes Immun 12: 149-154, 2011.

27. Ulivi P, Foschi G, Mengozzi M, Scarpi E, Silvestrini R, Amadori D and Zoli W: Peripheral Blood mir-328 expression as a potential biomarker for the early diagnosis of NSCLC. Int J Mol Sci 14: 10332-10342, 2013.

28. Arora S, Ranade AR, Tran NL, Nasser S, Sridhar S, Korn RL, Ross JT, Dhruv H, Foss KM, Sibenaller Z, et al: MicroRNA-328 is associated with (non-small) cell lung cancer (NSCLC) brain metastasis and mediates NSCLC migration. Int J Cancer 129: 2621-2631, 2011

29. Okumus NO, Gursel B, Meydan D, Ozdemir O, Odabas E and Gonullu G: Prognostic significance of concomitant radiotherapy in newly diagnosed glioblastomamultiforme: A multivariate analysis of 116 patients. Ann Saudi Med 32: 250-255, 2012.

30. Delic S, Lottmann N, Stelzl A, Liesenberg F, Wolter M, Götze S, Zapatka M, Shiio Y, Sabel MC, Felsberg J, et al: MiR-328 promotes glioma cell invasion via SFRP1-dependent Wnt-signaling activation. Neuro Oncol 16: 179-190, 2014.

31. Kozomara A and Griffiths-Jones S: miRBase: Annotating high confidence microRNAs using deep sequencing data. Nucleic Acids Res 42: D68-D73, 2014.

32. Brafman A, Mett I, Shafir M, Gottlieb H, Damari G, Gozlan-Kelner S, Vishnevskia-Dai V, Skaliter R, Einat P, Faerman A, et al: Inhibition of oxygen-induced retinopathy in RTP801-deficient mice. Invest Ophthalmol Vis Sci 45: 3796-3805, 2004.

33. Nidadavolu LS, Niedernhofer LJ and Khan SA: Identification of microRNAs dysregulated in cellular senescence driven by endogenous genotoxicstress. Aging (Albany NY) 5: 460-473, 2013

34. Huang JY, Chou SF, Lee JW, Chen HL, Chen CM, Tao MH and Shih C: MicroRNA-130a can inhibit hepatitis B virus replication via targeting PGC1 $\alpha$ and PPAR $\gamma$. RNA 21: 385-400, 2015.

35. Tawfik A, Sanders T, Kahook K, Akeel S, Elmarakby A and Al-Shabrawey M: Suppression of retinal peroxisome proliferator-activated receptor gamma in experimental diabetes and oxygen-induced retinopathy: Role of NADPH oxidase. Invest Ophthalmol Vis Sci 50: 878-884, 2009.

36. Simpson-Haidaris PJ, Pollock SJ, Ramon S, Guo N, Woeller CF, Feldon SE and Phipps RP: Anticancer role of PPARgamma agonists in hematological malignancies found in the vasculature, marrow and eyes. PPAR Res 2010: 814609, 2010. 